\title{
Rôle des optométristes de soins primaires dans l'évaluation et la prise en charge des patients ayant subi un traumatisme cérébral au Canada
}

Zoe Lacroix, D.O.

Résidente clinique, pédiatrie et thérapie visuelle École d'optométrie et de sciences de la vision, Université de Waterloo

Susan J. Leat, PhD, FCOptom, F.A.A.O. Professeure École d'optométrie et de sciences de la vision, Université de Waterloo

Lisa W. Christian, D.O., F.C.O.V.D., F.A.A.O.

Professeure agrégée de clinique École d'optométrie et de sciences de la vision, Université de Waterloo

\section{Introduction}

Les traumatismes cérébraux (TCC) résultent d'un choc violent à la tête qui perturbe le fonctionnement normal du cerveau. ${ }^{1}$ On catégorise le TCC en trois degrés de gravité qui vont de léger à grave, selon l'état mental du patient, son niveau de conscience et l'amnésie provoquée par la lésion. Selon une estimation prudente, l'incidence annuelle du TCC en Amérique du Nord et en Europe est d'environ 600/100 000..,3 Cela représente au moins 200000 cas de TCC au Canada chaque année. Selon les Centers for Disease Control and Prevention et l'Institut canadien d'information sur la santé, les principales causes de TCC entraînant une hospitalisation sont les chutes (35-45\%), suivies des accidents de la route (17 - $36 \%$ ), des événements liés aux collisions (se heurter sur ou être heurté par) (10 -17\%) et des agressions ( $9-10 \%)^{4,5}$ Les blessures à la tête sont plus courantes chez les enfants et les jeunes (0-19 ans), suivis par les personnes âgées (60 ans et plus). Ils sont aussi plus fréquents chez les hommes que chez les femmes, et ce, dans chaque groupe d'âge. Cependant, il convient de noter que la démographie des patients qui se présentent dans un cabinet d'optométriste peut différer de celle qui est basée sur les admissions à l'hôpital.

Les traumatismes cérébraux sont classés en fonction de la durée de la perte de conscience et de l'amnésie post-traumatique, ainsi que des résultats de l'imagerie cérébrale (Tableau 1). ${ }^{6,7}$ Tous ces signes ne sont pas nécessairement présents. Selon Menon et coll., un diagnostic de TCC peut être établi lorsqu'il y a altération de la fonction cérébrale définie par l'un des signes suivants : une période d'altération du niveau de conscience ou de perte de conscience, une perte de mémoire pour des événements survenus immédiatement avant ou après la blessure, des déficits neurologiques (faiblesse, perte d'équilibre, changement de la vision, parésie, paralysie ou dyspraxie, perte sensorielle, aphasie, etc.), et toute altération de l'état mental au moment de la blessure (confusion, désorientation, ralentissement de la pensée, etc.). ${ }^{8}$

Les phénomènes fréquents à la suite d'un TCC comprennent une diminution de l'attention, de la concentration et de la vitesse de traitement de l'information, des problèmes de mémoire, de la confusion, de l'irritabilité, de la dépression et de l'anxiété. Les conséquences physiques peuvent inclure des céphalées, de la fatigue, des étourdissements et des nausées, des troubles de l'équilibre, des troubles visuels et une perturbation du sommeil ${ }^{1}$. En cas de TCC modéré à grave, les patients peuvent également éprouver une baisse de la fonction exécutive, une confusion accrue, des symptômes de dépression, de l'anxiété, un manque de contrôle des impulsions, de la douleur chronique et des répercussions physiques graves. ${ }^{6}$ Des symptômes visuels sont observés dans $75 \%$ des cas de TCC. ${ }^{9}$ Ces symptômes peuvent être causés par une diminution de la fonction visuelle, des 
troubles du système de vision binoculaire, des changements dans la santé oculaire et des troubles du traitement plus complexes, qui seront présentés plus loin. En raison du large éventail de symptômes visuels qui peuvent se manifester après un traumatisme cérébral, il est important que l'optométriste de soins primaires soit familier avec les tests et la prise en charge des patients ayant des antécédents de lésion cérébrale traumatique.

Tableau 1 : Système de classification du $T C^{6,7}$

\begin{tabular}{|l|l|l|l|}
\hline Caractéristiques & TC léger & TC modéré & TC grave \\
\hline Perte de conscience & 0 à 30 minutes & 0,5 à 24 heures & $>24$ heures \\
\hline Amnésie post-traumatique (APT) & $0-1$ jour & $1-7$ jours & $>7$ jours \\
\hline Résultats de l'imagerie du cerveau & Normaux & Anormaux & Anormaux \\
\hline
\end{tabular}

\section{ÉVALUATION}

À la suite d'un TCC, une évaluation oculovisuelle complète doit être réalisée pour tous les patients. Les examens comprennent un historique détaillé des antécédents, la réfraction, la vision binoculaire et l'évaluation de l'accommodation courantes, des champs visuels automatisés et une évaluation de la santé oculaire avec un examen du fond d'œil sous mydriatique. L'évaluation oculovisuelle doit comprendre des tests supplémentaires approfondis de certains systèmes pour les patients avec un TCC suspecté ou diagnostiqué. Cela comprend des évaluations complémentaires de l'accommodation, de la vergence et du système oculomoteur, et peut comprendre des tests visuels de traitement de l'information et une évaluation du déplacement visuel de la ligne médiane.

Les personnes ayant subi un TCC sont également plus susceptibles de souffrir de troubles cognitifs et/ou de la mémoire, et il importe d'accorder une attention particulière à la vitesse à laquelle les tests sont administrés et à leur durée. Les patients qui ont subi un TCC ont souvent besoin de plus de temps pour traiter les questions et les indications. Par conséquent, les mesures objectives sont préférables, car elles fournissent souvent des résultats plus fiables. ${ }^{10}$ Les résultats des tests cliniques doivent comprendre tout problème d'étourdissements, de maux de tête, de nausées ou de photophobie qui aurait pu être signalé par le patient. Au besoin, il peut être préférable d'effectuer l'examen oculovisuel en deux étapes ou plus, réparties sur autant de rendez-vous.

\section{Antécédents médicaux}

Un examen approfondi des symptômes visuels devrait être effectué, ce qui peut être facilité en demandant au patient de remplir une liste de vérification des symptômes avant la consultation. ${ }^{11}$ Les antécédents médicaux devraient comprendre des renseignements sur l'incident responsable du TCC et les blessures associées. Il est utile d'examiner les services de réadaptation actuels et antérieurs du patient et les progrès de leur thérapie (ergothérapie, physiothérapie, etc.). Il faut également évaluer les objectifs et les besoins des patients, y compris les exigences visuelles liées à leur profession, à l'utilisation de l'ordinateur, à la conduite automobile, à la mobilité et à la lecture. Les optométristes doivent se rappeler d'inscrire au dossier les affections oculaires et l'état de santé général antérieurs (avant et après le TCC), afin d'établir une distinction entre les affections nouvelles et préexistantes.

\section{Acuité visuelle et réfraction}

Comme l'acuité visuelle elle-même est moins souvent affectée par le TCC, les méthodes habituelles (à savoir, le Snellen) peuvent être utilisées pour évaluer l'acuité visuelle chez les patients ayant subi un TCC. Si un patient est atteint de troubles cognitifs ou de la communication, l'utilisation d'un tableau optométrique Tumbling E ou d'un test Broken Wheel peuvent fournir des résultats plus fiables. ${ }^{11}$

Lorsque l'optométriste effectue un examen de la réfraction, il faut envisager de recourir à des méthodes de mesure objectives comme la rétinoscopie pour tous les patients, car il peut être difficile d'obtenir des réponses subjectives fiables. Il est également possible d'utiliser un réfracteur automatique pour les patients photophobes. Bien que les TCC ne modifient pas directement l'erreur de réfraction d'un patient, cette population peut devenir plus sensible aux petites modifications de prescription ou aux erreurs de réfraction non corrigées. Il faut accorder une attention particulière aux patients souffrant d'hypermétropie latente ou non corrigée, qui peuvent 
devenir symptomatiques à la suite d'un TCC. ${ }^{12}$ Les verres à foyer progressif ne sont pas recommandés en raison de distorsions périphériques.

\section{Santé oculaire}

Un examen approfondi à la lampe à fente est effectué pour évaluer la santé oculaire des patients ayant subi un TCC, y compris un examen du fond d'œil sous mydriatique. Les troubles oculaires consécutifs à un TCC peuvent toucher les segments antérieur et postérieur et peuvent comprendre une récession de l'angle, une sécheresse oculaire, une hémorragie intraoculaire ou des embolies et un odème papillaire. ${ }^{13-17}$ Une évaluation approfondie des nerfs crâniens, des pupilles et des nerfs optiques doit également être effectuée. La prise en charge de ces affections exige un traitement approprié ou, le cas échéant, l'aiguillage du patient vers un médecin de famille, un ophtalmologiste, un neuro-ophtalmologiste, un neurologue, etc.

\section{Champs visuels}

Les altérations du champ visuel peuvent survenir à la suite d'un traumatisme du nerf optique, du chiasma, des voies optiques ou du cortex occipital. ${ }^{15-17}$ Les altérations légères du champ visuel peuvent ne pas être détectées par un examen du champ visuel par confrontation. ${ }^{18}$ La périmétrie automatisée est mieux adaptée à la détection de légers troubles neurologiques et au suivi des changements au fil du temps. ${ }^{18}$ Le dépistage de la négligence visuelle devrait également être envisagé chez les patients ayant subi un TCC. ${ }^{19-21}$ Les tests d'inattention spatiale (négligence) comprennent le test de bissection de la ligne et le test du dessin de l'horloge. ${ }^{22}$

À la suite d'une évaluation adéquate de l'altération du champ visuel, les méthodes de traitement visent généralement à accroître la conscience du champ affecté et à développer des techniques de compensation. Ceci peut être réalisé en utilisant des prismes qui améliorent le champ tel que des prismes sectoriels ou des prismes de Peli. ${ }^{23,24}$ Ces prismes visent à mettre en évidence l'image du champ affecté afin de fournir au patient des renseignements sur sa périphérie. Des techniques de compensation fonctionnelle et de réadaptation comme le balayage de champ et les techniques oculomotrices, comportementales et de lecture peuvent également être enseignées aux patients. ${ }^{24,25}$

\section{Déplacement visuel de la ligne médiane}

Le syndrome du déplacement visuel de la ligne médiane (DVLM) a été défini comme une sensation de déplacement de l'égocentre et a été rapporté après des lésions cérébrales. ${ }^{26} \mathrm{Il}$ est souvent associé à la négligence et/ou à l'hémianopsie et peut même en résulter, bien que l'association exacte n'ait pas été documentée. Ces altérations dans la ligne médiane perçue peuvent entraîner des changements dans l'équilibre et la posture. Les professionnels de la santé qui traitent habituellement la démarche et l'équilibre comprennent les physiothérapeutes et les ergothérapeutes. ${ }^{26}$

Il n’y a pas de procédures d'évaluation normalisées pour le déplacement visuel de la ligne médiane. Les techniques actuelles comprennent l'alignement subjectif d'une baguette sur la ligne médiane, des essais de coordination œil-main, l'observation de la démarche, ainsi que des dispositifs émergents pour quantifier plus précisément la déviation de l'égocentre ${ }^{26}$. Padula et Argyris ont déclaré qu'un déplacement horizontal de la médiane a pour résultat une inclinaison latérale du côté opposé à l'espace visuel affecté, et une dérive possible vers la gauche ou la droite en marchant. Un déplacement vertical peut entraîner l'inclinaison du corps vers l'avant ou vers l'arrière (postérieure/antérieure). ${ }^{27}$

Bien qu’il soit nécessaire de poursuivre les recherches, certains praticiens ont rapporté avoir utilisé des «yoke prisms » compensatoires avec succès. ${ }^{26}$ Pour l'évaluation, la base des lentilles prismatiques est initialement dirigée vers le côté du déplacement perçu dans la ligne médiane pour réaligner l'égocentre du patient. L'essai est ensuite répété en utilisant d'autres lentilles. On procède généralement à ces essais avec des yoked prisms de moins de 10 à 12 dioptries prismatiques. Les thérapies de localisation spatiale ont également été utilisées pour améliorer la coordination œil-main. Une deuxième approche est l'adaptation prismatique, dans laquelle l'entraînement de localisation est entrepris avec des prismes dont la base est placée du côté opposé à la direction du déplacement. On utilise généralement des prismes plus puissants (17 dioptries prismatiques). Lorsque les prismes sont retirés, la fixation devient plus centrale, et l'amélioration peut durer jusqu'à 3,5 ans. ${ }^{28}$ 


\section{Accommodation}

Les troubles de l'accommodation sont présents chez environ $40 \%$ des patients ayant subi un TCC $\mathrm{TC}^{29,30}$ et comprennent une insuffisance d'accommodation, une difficulté d'accommodation ou des spasmes d'accommodation (qui peuvent induire une pseudo-myopie). ${ }^{31}$ L'examen de l'accommodation doit comprendre l'évaluation de l'amplitude d'accommodation (par le « push-up test » ou la courbe de défocalisation), de l'exactitude accommodative (méthode d'estimation de la vision monoculaire, méthode du cylindre croisé, ou rétinoscopie dynamique modifiée de Nott) et de la facilité d'accommodation (monoculaire et binoculaire).$^{10}$

La prise en charge des troubles de l'accommodation peut inclure des lunettes de lecture avec verres positifs ${ }^{10}$ ou des exercices de réadaptation de la vision. ${ }^{10,32,33}$ Chez les patients non presbytes, on recommande généralement des exercices oculaires comme traitement initial, qui peuvent comprendre des exercices d'alternance entre des lentilles ou distances différentes ainsi que des exercices de focale (accommodative push-up). Il y a quelques données probantes montrant que la thérapie visuelle améliore les troubles de l'accommodation chez $87-100 \%$ des patients. ${ }^{33}$

\section{Vision binoculaire}

Les troubles de vergence sont l'un des troubles les plus fréquents après un TCC et sont observés chez environ $50 \%$ des patients. ${ }^{9,29,34}$ Les troubles courants comprennent l'insuffisance de convergence (36 \%), l'instabilité binoculaire (étendue des vergences restreinte) (10\%), l'ésophorie (18\% des patients ayant subi un accident vasculaire cérébral) et le strabisme (p. ex., exotropie intermittente, paralysie du nerf crânien) (7-25 \%)., 9,29,34

L'examen de la vision binoculaire doit inclure des procédures courantes et des tests supplémentaires, y compris l'alignement oculaire à distance et de près (test de l'écran, baguette de Maddox, phorie, phorie associée), la fusion motrice (étendue des vergences, point de convergence proche, flexibilité de convergence avec alternance de prisme 3BI/12BO), la fusion sensorielle (stéréoscopie et fusion) et la motilité oculaire. ${ }^{12}$

La prise en charge des troubles de vergence peut inclure des verres, un prisme correcteur ou des exercices de thérapie visuelle. ${ }^{12,33,35}$ La thérapie visuelle est habituellement recommandée comme traitement initial de l'insuffisance de convergence, alors que les verres positifs devraient être pris en considération pour l'excès de convergence. L'entraînement en vision binoculaire en cabinet a été utilisé pour traiter avec succès plus de 75 \% des patients ayant subi un TCC qui présentent une insuffisance de convergence. ${ }^{33,35}$ Ces thérapies incluent les cordes de Brock, l'exercice du crayon, des alternances de prisme ou des instruments tels que le diploscope, le cheiroscope, les vectogrammes et les tranaglyphes, souvent en combinaison.

\section{Système oculomoteur}

La fixation, les poursuites et les saccades sont affectées chez environ $20 \%$ des patients ayant subi un TCC. ${ }^{36}$ Les procédures d'évaluation de la fonction oculomotrice incluent le Developmental Eye Movement Test, le King Devick Test, les lunettes Visagraph/ReadAlyzer avec capteurs infrarouges ainsi que les tests oculomoteurs NSUCO (Northeastern State University College of Optometry) et SCCO (Southern California College of Optometry).

Le traitement vise à exercer chacune de ces compétences individuelles. Certaines données montrent que les thérapies oculomotrices réussissent à améliorer ces compétences, en particulier en lecture. ${ }^{12,33,37}$ Bien que les techniques servant à améliorer les capacités oculomotrices n'aient pas fait l'objet de recherches approfondies, les thérapies peuvent inclure des cahiers de suivi des lettres, des exercices de poursuite oculomotrice, la fixation d'une corde de Brock, le cache-cache lumineux et des programmes informatisés (c.-à-d., Home Therapy System [HTS] ou un autre logiciel de thérapie visuelle assistée par ordinateur).

\section{Photophobie}

Les patients rapportent fréquemment une photophobie et une sensibilité accrue à l'éblouissement à la suite d'un TCC. ${ }^{38}$ Malgré sa prévalence, la photophobie demeure mal comprise et difficile à évaluer et à traiter. Des recherches sont en cours dans ce domaine pour mieux comprendre les mécanismes sous-jacents. Diverses théories ont attribué la photophobie aux migraines causées par le TCC, à des lésions de la structure intracrânienne sensible à la douleur et à des troubles d'adaptation à l'obscurité. ${ }^{88-40}$

Les antécédents médicaux des patients ayant subi un TCC doivent comprendre des questions sur l'augmentation de la sensibilité à l'éblouissement, à la lumière du soleil, aux ordinateurs et aux écrans. ${ }^{41}$ Des tests minutieux des 
pupilles doivent être effectués, bien que cela donne souvent des résultats normaux. La sécheresse oculaire et les céphalées devraient faire l'objet d'un examen approfondi, car ces problèmes peuvent exacerber les symptômes de photophobie. ${ }^{38}$ Toutes les maladies sous-jacentes doivent être traitées de manière appropriée.

Bien qu'aucune étude majeure n'ait été menée sur la prise en charge des symptômes de photophobie, les options de traitement actuelles comprennent des verres teintés, des filtres et des lunettes de soleil polarisées, photochromiques ou ajustables. ${ }^{41}$ Ces options sont généralement choisies de façon subjective, mais soulagent souvent les patients et améliorent leur confort visuel. Cependant, certaines données montrent que l'absence de teinte, les teintes plus claires ou une diminution graduelle de la teinte favorisent une diminution de la photosensibilité au fil du temps. ${ }^{42}$

\section{APPROCHE MULTIDISCIPLINAIRE}

Il n'est pas rare que les patients ayant subi un TCC présentent des problèmes de santé concomitants. Une approche multidisciplinaire est toujours recommandée lors de la prise en charge de ces patients. La collaboration interprofessionnelle avec d'autres fournisseurs de soins de santé permet d'améliorer les soins aux patients grâce à des rapports d'étape et des communications réguliers. En plus des optométristes et des ophtalmologistes, les autres spécialistes qui s'occupent souvent des patients ayant subi un TCC comprennent les médecins, les neurologues, les physiothérapeutes, les ergothérapeutes, les audiologistes, les spécialistes en thérapie vestibulaires, les physiothérapeutes et les chiropraticiens. Il est recommandé que les optométristes développent une bonne relation avec les autres fournisseurs afin de garantir des soins optimaux aux patients. Lorsque d'autres professionnels de la santé partagent la prise en charge du patient, ils devraient recevoir un rapport présentant les résultats de l'examen oculovisuel et les recommandations concernant ces patients.

\section{CONCLUSION}

Comme nous venons de le voir, les symptômes visuels sont très fréquents après un TCC. Ces patients tirent avantage d'une évaluation optométrique approfondie visant à identifier et à prendre en charge tout problème de vision sous-jacent. Les traitements peuvent comprendre des lentilles teintées et des filtres, des verres correcteurs et prismatiques, ainsi que la thérapie et la rééducation visuelle. En répondant aux besoins visuels des patients ayant subi un TCC, nous pouvons réduire leurs symptômes, améliorer leur qualité de vie et les aider à retourner au travail et à la vie quotidienne. $\bullet$

\section{RÉFÉRENCES}

1. Centers for Disease Control and Prevention (CDC), National Center for Injury Prevention and Control. Report to Congress on mild traumatic brain injury in the United States: steps to prevent a serious public health problem. Centers for Disease Control and Prevention. 2003.

2. Cassidy J.D., Carroll L.J., Peloso P.M., et coll. Incidence, risk factors and prevention of mild traumatic brain injury: results of the WHO Collaborating Centre Task Force on Mild Traumatic Brain Injury. J Rehabil Med. 2004(43 Suppl):28-60.

3. Instituts de recherche en santé du Canada. Recherche sur les lésions cérébrales traumatiques [Disponible à l'adresse : http://www. cihr-irsc.gc.ca/f/45665.html]. Consulté le 2017-01-05.

4. Centers for Disease Control and Prevention. Traumatic brain injury in the United States: emergency department visits, hospitalizations, and deaths, 2002-2006. Atlanta (GA); 2010.

5. L'Institut canadien d'information sur la santé Traumatismes crâniens au Canada : Une décennie de changements (1994-1995 à 2003-2004). 2006. [Disponible à l'adresse : https://secure.cihi. ca/free_products/ntr_head_injuries_2006_f.pdf]. Consulté le 2017-01-05.

6. Centers for Disease Control and Prevention. Injury Prevention \& Control: Traumatic Brain Injury \& Concussion.

7. Blyth B.J., Bazarian J.J. Traumatic alterations in consciousness: traumatic brain injury. Emerg Med Clin North Am. 2010;28(3):571-94.

8. Menon D.K., Schwab K., Wright D.W., Maas A.I. Énoncé de position : Definition of traumatic brain injury. Arch Phys Med Rehab 2010;91(11):1637-40.

9. Brahm K.D., Wilgenburg H.M., Kirby J., Ingalla S., Chang C.Y., Goodrich G.L. Visual impairment and dysfunction in combatinjured servicemembers with traumatic brain injury. Optom Vis Sci. 2009;86(7):817-25
10. Cohen A. Acquired visual information-processing disorders: closed head trauma. Applied Concepts in Vision Therapy, Press L, éd. St Louis: Mosby; 1997. p. 154-66.

11. Kara G., Chrystyna R. Examination Approach. 2013. Dans : Brain Injury Electronic Resource Manual [Disponible à l'adresse : http:// aoa.uberflip.com/i/225867-brain-injury-electronic-resource-manual]. American Optometric Association Vision Rehabilitation Section. Consulté le 2017-01-05.

12. Scheiman M., Wick B. Binocular and Accommodative Problems Associated with Acquired Brain Injury. Dans : Clinical Management of Binocular Vision: Heterophoric, Accommodative and Eye Movement Disorders, 2nd ed. Philadelphia, PA: Lippincott, Williams and Wilkins; 2002, p. 571-592.

13. Cockerham G.C., Lemke S., Glynn-Milley C., Zumhagen L., Cockerham K.P. Visual performance and the ocular surface in traumatic brain injury. Ocul Surf 2013;11 (1):25-34.

14. Candice E. Ocular Health Examination. 2013. Dans : Brain Injury Electronic Resource Manual [Disponible à l'adresse : http://aoa. uberflip.com/i/225867-brain-injury-electronic-resource-manual]. American Optometric Association Vision Rehabilitation Section. Consulté le 2017-01-05.

15. Atkins E.J., Newman N.J., Biousse V. Post-traumatic visual loss. Rev Neurol Dis 2008;5(2):73-81.

16. Jacobs S.M., Van Stavern G.P. Neuro-ophthalmic deficits after head trauma. Curr Neurol Neurosci Rep 2013;13(11):389.

17. Cockerham G.C., Goodrich G.L., Weichel E.D., et coll. Eye and visual function in traumatic brain injury. J Rehabil Res Dev 2009;46(6):811-8.

18. Kerr N.M., Chew S.S., Eady E.K., Gamble G.D., Danesh-Meyer H.V. Diagnostic accuracy of confrontation visual field tests. Neurology 2010;74(15):1184-90.

19. Goodrich G.L., Kirby J., Cockerham G., Ingalla S.P., Lew H.L. Visual function in patients of a polytrauma rehabilitation center: A 
descriptive study. J Rehabil Res Dev 2007;44(7):929-36.

20. Halterman C.I., Langan J., Drew A., et coll. Tracking the recovery of visuospatial attention deficits in mild traumatic brain injury. Brain 2006;129(3):747-53.

21. Suchoff I.B., Ciuffreda K.J. A primer for the optometric management of unilateral spatial inattention. Optometry 2004;75(5):305-18.

22. Kara G., Chrystyna R., Brenda M., Michael P. Examination of Cortical Visual Function. 2013. Dans : Brain Injury Electronic Resource Manual [Disponible à l'adresse : http://aoa.uberflip.com/i/ 225867brain-injury-electronic-resource-manual]. American Optometric Association Vision Rehabilitation Section. Consulté le 2017-01-05.

23. Ross N.C., Bowers A.R., Peli E. Peripheral prism glasses: effects of dominance, suppression, and background. Optom Vis Sci 2012;89(9):1343-52.

24. Kapoor N., Ciuffreda K.J. Vision disturbances following traumatic brain injury. Curr Treat Options Neurol 2002;4(4):271-80.

25. Goodwin D. Homonymous hemianopia: challenges and solutions. Clin Ophthalmol (Auckland, NZ). 2014; 8:1919-27. (en anglais)

26. Padula W.V., Nelson C.A., Benabib R., Yilmaz T., Krevisky S. Modifying postural adaptation following a CVA through prismatic shift of visuo-spatial egocenter. Brain Inj 2009;23(6):566-76.

27. Padula W.V., Argyris S. Post trauma vision syndrome and visual midline shift syndrome. NeuroRehabilitation 1996;6(3):165-71.

28. Keane S., Turner C., Sherrington C., Beard J.R. Use of fresnel prism glasses to treat stroke patients with hemispatial neglect. Arch Phys Med Rehabil 2006;87:1668-72.

29. Ciuffreda K.J., Kapoor N., Rutner D., Suchoff I.B., Han M.E., Craig S. Occurrence of oculomotor dysfunctions in acquired brain injury: a retrospective analysis. Optometry 2007;78(4):155-61.

30. Green W., Ciuffreda K.J., Thiagarajan P., Szymanowicz D., Ludlam D.P., Kapoor N. Accommodation in mild traumatic brain injury. $J$ Rehabil Res Dev 2010;47(3):183-99.
31. London R., Wick B., Kirschen D. Post-traumatic pseudomyopia. Optometry 2003;74(2):111-20.

32. Scheiman M., Cotter S., Kulp M.T., et coll. Treatment of accommodative dysfunction in children: results from a randomized clinical trial. Optom Vis Sci 2011;88(11):1343-52.

33. Ciuffreda K.J., Rutner D., Kapoor N., Suchoff I.B., Craig S., Han M.E. Vision therapy for oculomotor dysfunctions in acquired brain injury: a retrospective analysis. Optometry 2008;79(1):18-22.

34. Capo-Aponte J.E., Urosevich T.G., Temme L.A., Tarbett A.K., Sanghera N.K. Visual dysfunctions and symptoms during the subacute stage of blast-induced mild traumatic brain injury. Mil Med 2012;177(7):804-13.

35. Scheiman M., Mitchell G.L., Cotter S., et coll. A randomized clinical trial of treatments for convergence insufficiency in children. Arch Ophthalmo. 2005;123(1):14-24.

36. Hunt A.W., Mah K., Reed N., Engel L., Keightley M. Oculomotorbased vision assessment in mild traumatic brain injury: a systematic review. J Head Trauma Rehabil 2016;31(4):252-61.

37. Ciuffreda K.J., Han Y., Kapoor N., Ficarra A.P. Oculomotor rehabilitation for reading in acquired brain injury. NeuroRehabilitation 2006;21(1):9-21.

38. Digre K.B., Brennan K.C. Shedding light on photophobia. J Neuroophthalmol 2012;32(1):68-81.

39. Du T., Ciuffreda K.J., Kapoor N. Elevated dark adaptation thresholds in traumatic brain injury. Brain Inj 2005;19(13):1125-38.

40. Nampiaparampil D.E. Prevalence of chronic pain after traumatic brain injury: a systematic review. JAMA 2008;300(6):711-9.

41. Barnett B., Singman E. Vision concerns after mild traumatic brain injury. Curr Treat Options Neurol 2015.17(2):329.

42. Truong J.Q., Ciuffreda K.J., Han M.H., Suchoff I.B. Photosensitivity in mild traumatic brain injury (mTBI): A retrospective analysis. Brain Inj 2014;28(10):1283-7.

\section{HELP YOUR PATIENTS DISCOVER NEW VISION CARE OPTIONS}

Our Co-Management program offers many advantages, including:

- Access to any of our 30+ clinics across Canada

- A standardized Co-Management program

- Co-Management team available to facilitate patient care coordination

- An experienced team of surgeons who have collectively performed over 1 million procedures*

- Affordable pricing starting at $\$ 490$ per eye*

To book a free consultation for your patient, contact us directly at comanagement@lasikmd.com

\section{LASIKMD}

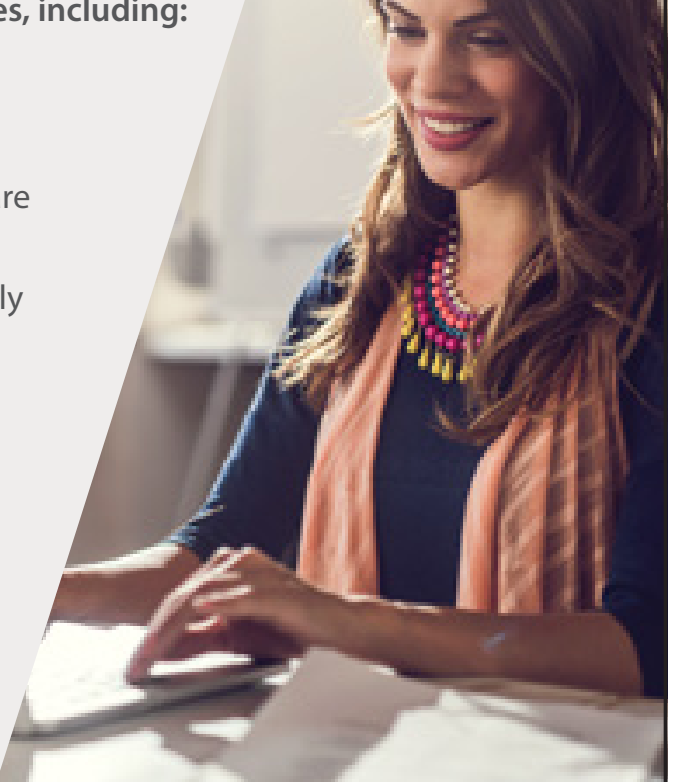

*Including 425,000 procedures in Quebec alone. ${ }^{* *}$ Prices are subject to change without prior notice and vary based on prescription strength. Standard LASIK starting at $\$ 490 /$ eye. Other conditions may apply. 